\title{
WHAT IS THE JACOBIAN OF A RIEMANN SURFACE WITH BOUNDARY?
}

\author{
THOMAS M. FIORE AND IGOR KRIZ
}

\begin{abstract}
We define the Jacobian of a Riemann surface with analytically parametrized boundary components. These Jacobians belong to a moduli space of "open abelian varieties" which satisfies gluing axioms similar to those of Riemann surfaces, and therefore allows a notion of "conformal field theory" to be defined on this space. We further prove that chiral conformal field theories corresponding to even lattices factor through this moduli space of open abelian varieties.
\end{abstract}

\section{INTRODUCTION}

The main purpose of the present note is to generalize the notion of the Jacobian of a Riemann surface to Riemann surfaces with real-analytically parametrized boundary (or, in other words, conformal field theory worldsheets). The Jacobian of a closed surface is an abelian variety. What structure of "open abelian variety" captures the relevant data in the "Jacobian" of a CFT worldsheet? If we considered Riemann surfaces with punctures instead of parametrized boundary components, the right answer could be easily phrased in terms of mixed Hodge structures.

But in worldsheets, we see more structure, and some of it is infinitedimensional. For example, even to a disk with analytically parametrized boundary, one naturally assigns an infinite-dimensional symplectic form and a restricted maximal isotropic space (cf. [7]). Any structure we propose should certainly include such data. Additionally, in worldsheets, boundary components can have inbound or outbound orientation, and an inbound and outbound boundary component can be glued to produce another worldsheet. So another test of having the right notion of "open abelian variety" is that it should enjoy a similar gluing structure.

The first author was supported at the University of Chicago by NSF Grant DMS0501208. At the Universitat Autònoma de Barcelona he was supported by Grant SB20060085 of the Programa Nacional de ayudas para la movilidad de profesores de universidad e investigadores españoles y extranjeros. The second author was supported in part by NSF Grant DMS-0305583. 
We should point out that it is actually a remarkably strong requirement that a structure such as a (closed) abelian variety could somehow be "glued together" from "genus 0" data similar to the situation we described above for a disk. One quickly convinces oneself that naive approaches based on modelling somehow the 1-forms on a Riemann surface, together with mixed Hodge-type integral structure data, fail to produce the required gluing. In fact, in some sense, the desired structure must be "pure" rather than "mixed". Note that there is no way of "gluing" a pure Hodge structure out of a mixed Hodge structure which does not already contain it: in the case of a closed Riemann surface with punctures, the mixed Hodge structure on its first cohomogy contains the pure Hodge structure of the original closed surface, so no gluing is involved. Clearly, the situation is different when we are gluing a non-zero genus surface from a genus 0 surface with parametrized boundary.

There is, however, a yet stronger test. When $L$ is an even lattice (together with a $\mathbb{Z} / 2$-valued bilinear form $b$ satisfying a suitable condition), one has a notion of conformal field theory associated with $L([9,4])$. It could be argued that the definition only uses additive data, so the lattice conformal theories should "factor through open abelian varieties". In some sense, if one considers the conjectured space of open abelian varieties to be the "Jacobian" of the moduli space of worldsheets (with all its structure), then one could interpret this as a sort of "Abelian Langlands correspondence" for that space. This test is also severe, as lattice conformal field theories are known to be unexpectedly tricky. For example, the definition of operator assigned to a worldsheet appears to depend on the order of boundary components, and a subtle discussion is needed to remove this (unacceptable) dependency. This will be clarified in Section 5 below.

In this paper, we indeed propose a notion of an open abelian variety and answer both test questions in the affirmative. Of course, one has to start out by being precise about what exact abstract structure captures the notion of gluing, and then generalize the notion of conformal field theory to be defined on such abstract structures. Following ideas of Segal [9], this was done in $[1,4,5]$, with a correction in [3]. The desired structure is called stack of pseudo commutative monoids with cancellation (SPCMC - see [3] for a correct definition) and a CFT is a pseudo morphism of certain SPCMC's

$$
\mathcal{C} \rightarrow C(\mathcal{M}, \mathcal{H})
$$


(The papers $[4,5]$ used the word "lax" instead of "pseudo", but the first author [1] discovered that "pseudo" conforms more with existing terminology of higher category theory. $)^{1}$

In the present paper, the meaning of the target of the map (1), which is defined in [5], plays only a marginal role. The source of the map (1), however, is important: it is the SPCMC of Segal's worldsheets. Those are 2-dimensional real-analytic manifolds with boundary which have a complex structure and real-analytically parametrized boundary components. The notion of SPCMC, which is defined in $[4,5]$, is designed to capture the operations of disjoint union and gluing in $\mathcal{C}$, along with the fact that $\mathcal{C}$ is a groupoid (under holomorphic maps compatible with the boundary parametrizations), and in fact a stack over the Grothendieck topology of complex-analytic manifolds and open covers. In particular, gluing in $\mathcal{C}$ is defined by noticing that the parametrized boundary components of a worldsheet can have two possible orientations with respect to the complex structure - one usually calls them inbound and outbound. Now from a worldsheet $X$, another worldsheet, usually denoted by $X^{\nabla}$ (despite of the ambiguity of the symbol), can be obtained by gluing an inbound boundary component of $X$ to an outbound, using the parametrizations. The notion of SPCMC is designed to capture all the algebraic properties of these operations.

The definition (1) may seem mysterious, but roughly speaking, we can imagine we have a certain finite set of labels $A$, Hilbert spaces $H_{a}$ for $a \in A$, and for every worldsheet $X$ with a map $\phi$ assigning to each boundary component $c$ of $X$ a label $\phi(c) \in A$, a finite-dimensional vector space $M_{X, \phi}$ and a trace class element

$$
U_{X, \phi} \in M_{X, \phi} \otimes \hat{\bigotimes}_{c} H_{\phi(c)}^{*} \hat{\otimes} \hat{\otimes} H_{\phi(d)}
$$

where the tensor products are over inbound boundary components $c$ and outbound boundary components $d$ of $X$. The symbol $\hat{\otimes}$ means Hilbert tensor product. These elements (called vacuum elements) are required to satisfy certain properties which we will not list here. However, one important example is in order. When $X$ has no boundary components (is a closed surface), (2) becomes simply an element of $M_{X}$ ( $\phi$ is dummy), and it follows from the structure that $M_{X}$ is a representation of the mapping class group $\operatorname{Mod}(X)$.

\footnotetext{
${ }^{1}$ It should be pointed out that instead of SPCMC's, we could use other known structures present on worldsheets which can be used for axiomatizing CFT, for example the 'cobordism approach' based on PROPs; our structure satisfies those axioms as well. As shown in [2], however, when one carefully treats the cobordism approach so no relevant axioms are omitted, the discussion is comparable to SPCMC's.
} 
However, physicists noticed that in some cases (e.g. the lattice theories) more is true, namely that the representation of the mapping class group $\operatorname{Mod}(X)$ on $M_{X}$ extends to the Siegel modular group $S p(2 g, \mathbb{Z})$ where $g$ is the genus of $X$ (there is a natural map $\operatorname{Mod}(X) \rightarrow S p(2 g, \mathbb{Z})$ by taking 1 st cohomology). The question therefore arises: what does it mean for a CFT to be "Siegel-modular", or, in other words, to depend only on the cohomology of the worldsheet $X$ ?

It is the main purpose of this note to provide one possible answer to this question. Our approach is to define a pseudo morphism of SPCMC's

$$
\mathcal{C} \rightarrow \mathcal{J}
$$

where $\mathcal{J}$ is, roughly speaking, the SPCMC of all possible "structures that look like cohomologies of worldsheets". We define precisely what this means, and call such structures 'open abelian varieties'.

Defining the SPCMC of open abelian varieties is our main result. We also show that the (chiral) lattice CFT corresponding to an even lattice indeed factors through a CFT on $\mathcal{J}$ by the map (3), which explains its Siegel modularity. The reader is invited to notice that such a discussion would be very difficult, if not impossible, if the notion of SPCMC were not developed.

The present paper is organized as follows. In Section 2, we define open abelian varieties, and discuss their moduli stack. In Section 3, we discuss gluing of open abelian varieties, and their SPCMC structure. In Section 4, we discuss the Jacobian map from the SPCMC of worldsheets to the SPCMC of open abelian varieties. In Section 5 , we shall discuss the lattice conformal field theory on the SPCMC of open abelian varieties.

\section{OPEn ABELIAN VARIETIES}

Construction 2.1. Let us start with the space $V_{1}$ of real-analytic functions

$$
f: \mathbb{R} \rightarrow \mathbb{R}
$$

for which there exists a number $\Delta_{f}$ such that

$$
f(x+2 \pi)=f(x)+\Delta_{f} .
$$

We may then alternately think of $V_{1}$ as a space of "branched" functions on $S^{1}$ by applying the map $e^{i z}$. There is an antisymmetric form $S$ on $V_{1}$ given by

$$
S(f, g)=\int_{S^{1}} f d g-\Delta_{f} g(0)-\frac{1}{2} \Delta_{f} \Delta_{g}
$$


(the integral over $S^{1}$ is interpreted as the integral from 0 to $2 \pi$ ). Note that in (4), the term $\Delta_{f} g(0)$ could have been equally well replaced by $\Delta_{g} f(0)$. The point is to choose the terms so that $S(f, g)=-S(g, f)$.

Given a pair of disjoint finite sets $A^{+}$and $A^{-}$, we set

$$
A=A^{+} \amalg A^{-} \text {. }
$$

(We think of $A^{+}$as the set of outbound and $A^{-}$as the set of inbound boundary components within a connected component.) Define

$$
V_{A}=\left\{f=\left(f_{i}\right)_{i} \in \prod_{i \in A^{+} \amalg A^{-}} V_{1} \mid \sum_{i \in A^{+}} \Delta_{f_{i}}-\sum_{i \in A^{-}} \Delta_{f_{i}}=0\right\} /\left\langle(1)_{i}\right\rangle .
$$

Now choose a linear ordering on the set $A$. For $i \in A$, define $\epsilon_{i}=1$ if $i \in A^{+}$ and $\epsilon_{i}=-1$ if $i \in A^{-}$. Define for $f, g \in V_{A}$,

$$
S_{A}(f, g)=\sum_{i \in A} \epsilon_{i} S\left(f_{i}, g_{i}\right)-\frac{1}{2} \sum_{i<j \in A} \epsilon_{i} \epsilon_{j}\left(\Delta_{f_{i}} \Delta_{g_{j}}-\Delta_{g_{i}} \Delta_{f_{j}}\right) .
$$

Note that since the space $V_{A}$ is fixed, we can also give it an integral structure, i.e. a topological basis $B_{A}$ on which $S_{A}$ is hyperbolic.

The exact choice does not matter. Note also that although the form (5) depends on the ordering of $A$, the antisymmetric forms $S_{<}$for different orderings $<$ are easily calculated from each other, by adding differences of the corresponding terms

$$
\frac{1}{2} \sum_{i<j} \epsilon_{i} \epsilon_{j}\left(\Delta_{f_{i}} \Delta_{g_{j}}-\Delta_{g_{i}} \Delta_{f_{j}}\right)
$$

Instead of speaking of an ordering and an antisymmetric form, it will be more useful for us to speak of a collection of antisymmetric forms $S_{<}$related to each other by the said formulas. We shall speak of antisymmetric forms $S_{<}$related in the standard way.

Remark 2.2. It will be important in the sequel to note that the form $S_{<}$in fact only depends on the cyclic ordering, i.e. if we take the smallest element 1 of $A$ and make it the greatest element without changing the order of the other elements, then the form $S$ does not change. To see this, note that the operation just described results in adding to $S$ the term

$$
\sum_{1 \neq j \in A} \epsilon_{1} \epsilon_{j}\left(\Delta_{f_{1}} \Delta_{g_{j}}-\Delta_{g_{1}} \Delta_{f_{j}}\right)
$$


But we are also assuming

$$
\sum_{j \in A} \epsilon_{j} \Delta_{f_{j}}=0=\sum_{j \in A} \epsilon_{j} \Delta_{g_{j}}
$$

so $(7)$ is equal to

$$
\epsilon_{1}^{2}\left(\Delta_{f_{1}} \Delta_{g_{1}}-\Delta_{g_{1}} \Delta_{f_{1}}\right)=0
$$

Remark 2.3. There is another way of relating the forms $S_{<}, S_{<^{\prime}}$ for different orders $<,<^{\prime}$ which will be of importance to us. Consider functions $f=\left(f_{i}\right)_{i}$ and $g=\left(g_{i}\right)_{i}$ as above. Then define

$$
f_{i}^{\prime}=f_{i}-\sum\left\{\epsilon_{j} \Delta_{f_{j}} \mid j<i \text { and } i<^{\prime} j\right\}
$$

We will refer to the map $f \mapsto f^{\prime}$ given by (9) as the standard transformation

$$
V_{A} \stackrel{\cong}{\longrightarrow} V_{A}
$$

corresponding to the change of the order $<$ to $<^{\prime}$. The relation we have in mind is established by the following result.

Lemma 2.4. We have

$$
S_{<}(f, g)=S_{<^{\prime}}\left(f^{\prime}, g^{\prime}\right)
$$

Proof: We have

$$
\begin{gathered}
S_{<}(f, g)=\sum_{i \in A} \epsilon_{i}\left(\int_{S^{1}} f_{i} d g_{i}-\Delta_{f_{i}} g_{i}(0)-\frac{1}{2} \Delta_{f_{i}} \Delta_{g_{i}}\right) \\
-\frac{1}{2} \sum_{i<j} \epsilon_{i} \epsilon_{j}\left(\Delta_{f_{i}} \Delta_{g_{j}}-\Delta_{g_{i}} \Delta_{f_{j}}\right)
\end{gathered}
$$




$$
\begin{aligned}
& =\sum_{i \in A} \epsilon_{i}\left(\int_{S^{1}} f_{i}^{\prime} d g_{i}^{\prime}+\sum_{j<i, i<<^{\prime} j} \int_{S^{1}} \epsilon_{j} \Delta_{f_{j}} d g_{i}^{\prime}-\Delta_{f_{i}^{\prime}}\left(g_{i}^{\prime}(0)+\sum_{j<i, i<j} \epsilon_{j} \Delta_{g_{j}}\right)\right. \\
& \left.-\frac{1}{2} \Delta_{f_{i}^{\prime}} \Delta_{g_{i}^{\prime}}\right)-\frac{1}{2} \sum_{i<j} \epsilon_{i} \epsilon_{j}\left(\Delta_{f_{i}^{\prime}} \Delta_{g_{j}^{\prime}}-\Delta_{g_{i}^{\prime}} \Delta_{f_{j}^{\prime}}\right) \\
& =\sum_{i \in A} \epsilon_{i}\left(\int_{S^{1}} f_{i}^{\prime} d g_{i}^{\prime}-\Delta_{f_{i}^{\prime}} g_{i}^{\prime}(0)-\frac{1}{2} \Delta_{f_{i}^{\prime}} \Delta_{g_{i}^{\prime}}\right) \\
& +\sum_{i \in A} \epsilon_{i}\left(\sum_{j<i, i<<^{\prime} j} \epsilon_{j} \Delta_{f_{j}^{\prime}} \Delta_{g_{i}^{\prime}}\right)-\sum_{i \in A} \epsilon_{i} \Delta_{f_{i}^{\prime}}\left(\sum_{j<i, i<<^{\prime} j} \epsilon_{j} \Delta_{g_{j}^{\prime}}\right) \\
& -\frac{1}{2} \sum_{i<j} \epsilon_{i} \epsilon_{j}\left(\Delta_{f_{i}^{\prime}} \Delta_{g_{j}^{\prime}}-\Delta_{g_{i}^{\prime}} \Delta_{f_{j}^{\prime}}\right) \\
& =\sum_{i \in A} \epsilon_{i} S\left(f_{i}^{\prime}, g_{i}^{\prime}\right) \\
& +\sum_{j<i, i<<^{\prime} j} \epsilon_{i} \epsilon_{j}\left(\Delta_{f_{j}^{\prime}} \Delta_{g_{i}^{\prime}}-\Delta_{g_{j}^{\prime}} \Delta_{f_{i}^{\prime}}\right)-\frac{1}{2} \sum_{i<j} \epsilon_{i} \epsilon_{j}\left(\Delta_{f_{i}^{\prime}} \Delta_{g_{j}^{\prime}}-\Delta_{g_{i}^{\prime}} \Delta_{f_{j}^{\prime}}\right) \\
& =\sum_{i \in A} \epsilon_{i} S\left(f_{i}^{\prime}, g_{i}^{\prime}\right) \\
& -\frac{1}{2} \sum_{j<i, i<<^{\prime} j} \epsilon_{i} \epsilon_{j}\left(\Delta_{f_{i}^{\prime}} \Delta_{g_{j}^{\prime}}-\Delta_{g_{i}^{\prime}} \Delta_{f_{j}^{\prime}}\right)+\frac{1}{2} \sum_{i<j, j<^{\prime} i} \epsilon_{i} \epsilon_{j}\left(\Delta_{f_{i}^{\prime}} \Delta_{g_{j}^{\prime}}-\Delta_{g_{i}^{\prime}} \Delta_{f_{j}^{\prime}}\right) \\
& -\frac{1}{2} \sum_{i<j}\left(\Delta_{f_{i}^{\prime}} \Delta_{g_{j}^{\prime}}-\Delta_{g_{i}^{\prime}} \Delta_{f_{j}^{\prime}}\right) \\
& =\sum_{i \in A} \epsilon_{i} S\left(f_{i}^{\prime}, g_{i}^{\prime}\right)-\frac{1}{2} \sum_{i<^{\prime} j} \epsilon_{i} \epsilon_{j}\left(\Delta_{f_{i}^{\prime}} \Delta_{g_{j}^{\prime}}-\Delta_{g_{i}^{\prime}} \Delta_{f_{j}^{\prime}}\right) \\
& =S_{<^{\prime}}\left(f^{\prime}, g^{\prime}\right) \text {. }
\end{aligned}
$$

It is also of interest to us that when $<^{\prime}$ is obtained from $<$ by moving the greatest element $i$ to the lowest, then $f_{j}^{\prime}=f_{j}$ for $j \neq i$, and $f_{i}^{\prime}$ is obtained from $f_{i}$ by adding the constant function equal to $\epsilon_{i} \Delta_{f_{i}}$. This 
means that when $<$ and $<^{\prime}$ correspond to the same cyclic order, the standard transformation is not necessarily the identity, but is given by adding to each $f_{i}$ a constant function which is a fixed integral multiple of $\Delta_{f_{i}}$.

Definition 2.5. An open abelian variety $\left(C, U, S, W, \iota, V_{\mathbb{Z}}^{\perp}\right)$ consists of a (possibly empty) set $C$ of finite sets (called open connected components) $A=A^{+} \amalg A^{-}$(whose elements are called outbound and inbound boundary components respectively), a real vector space $U$ with, for each system of linear orders $<$ of each $A \in C$, an embedding

$$
V:=\bigoplus_{A \in C} V_{A} \stackrel{\iota<}{\subseteq} U
$$

such that the image $\iota_{<} V$ is of finite codimension. (Note that the image $\iota_{<} V$ does not depend on $<$.) Further, for different choices of orders $<$ and $<^{\prime}$, the embeddings $\iota_{<}$and $\iota_{<^{\prime}}$ are related by composing with the standard transformation (see Remark 2.3). Further, a nondegenerate real symplectic form $S$ is given on $U$, and (10) maps the form

$$
\bigoplus_{A \in C} S_{<} \text {on } \bigoplus_{A \in C} V_{A}
$$

to $S$. (Note that by Remark 2.3 , it suffices to verify this assumption for one $\iota<$.)

Next, there is given a smooth (in the standard sense, see below) complex isotropic subspace $W \subset U_{\mathbb{C}}$ such that

$$
\begin{gathered}
W \oplus \bar{W}=U_{\mathbb{C}}, \\
2 i S(x, \bar{x})>0 \text { for all } x \in W
\end{gathered}
$$

(here $\bar{W}$ denotes the complex conjugate of $W$ ).

Additionally, there is an integral structure, which is the following subtle data: First, there is an integral structure on the $S$-complement $V^{\perp}$ (=annihilator) of $\iota_{<} V$, which means there is a subgroup $V_{\mathbb{Z}}^{\perp}$ of $V^{\perp}$ on which $S$ is isomorphic (but not by a given isomorphism) to a hyperbolic antisymmetric form.

Next, we impose an identification on open abelian varieties according to the following rule. Denote by $V_{\text {const }, \mathbb{Z}}$ the subgroup of $V$ consisting of functions which are constant, and have integral value, on every boundary component. Similarly, let $V_{d e g, \mathbb{Z}}$ denote the subspace of $V$ of functions which have integral degree on each boundary component, i.e. $\Delta_{f_{j}} \in \mathbb{Z}$ for all $j \in A \in C$. Fix a system of linear orders $<$ on each $A \in C$. Then two 
open abelian varieties $\left(C_{1}, U_{1}, S_{1}, W_{1}, \iota_{1}, V_{\mathbb{Z}, 1}^{\perp}\right)$ and $\left(C_{2}, U_{2}, S_{2}, W_{2}, \iota_{2}, V_{\mathbb{Z}, 2}^{\perp}\right)$ are identified if $C_{1}=C_{2}, U_{1}=U_{2}, S_{1}=S_{2}, W_{1}=W_{2}$ and the selection of the map $\iota_{<}$and $V_{\mathbb{Z}}^{\perp}$ is subject to the following rules: We require

$$
\begin{gathered}
V_{\mathbb{Z}, 1}^{\perp} \subseteq V_{\mathbb{Z}, 2}^{\perp} \oplus \iota_{2} V_{\text {const }, \mathbb{Z}}, \\
\left(\iota_{1}-\iota_{2}\right)\left(V_{\text {deg }, \mathbb{Z}}\right) \subseteq V_{\mathbb{Z}, 2}^{\perp} \oplus \iota_{2} V_{\text {const }, \mathbb{Z}} .
\end{gathered}
$$

Note that $\iota_{1}-\iota_{2}$ is a homomorphism. (Note that condition (14) implies that $\iota_{1}-\iota_{2}$ on $V$ only depends on the degree, as it is determined by its restriction to $V_{d e g, \mathbb{Z}}$, and the target of that map is discrete. It then follows

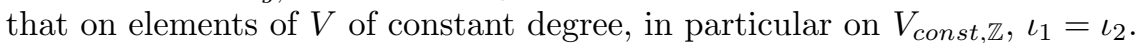
Because of this, one can replace $\iota_{2}$ by $\iota_{1}$, and/or $V_{\mathbb{Z}, 1}^{\perp}$ by $V_{\mathbb{Z}, 2}^{\perp}$ in (13). Also, because of this and (13), we may replace $\iota_{2}$ by $\iota_{1}$ and/or $V_{\mathbb{Z}, 2}^{\perp}$ by $V_{\mathbb{Z}, 1}^{\perp}$ on the right hand side of (14).)

Note also that by Remark 2.3, the choice of $<$ does not matter in this identification, since the identification is invariant under standard transformation. Note also that by the same remark, fixing $<$, we may replace $\iota_{<}$ by $\iota_{<}$, for any system of orders $<^{\prime}$ which defines the same cyclic order on each $A \in C$ without changing the open abelian variety.

To define smoothness of a subspace $W$, recall that we have a standard polarization of $V_{\mathbb{C}}$ given by the isotropic subspaces $V^{+}, V^{-}$of functions on each copy of $S^{1}$ which holomorphically (resp. antiholomorphically) extend to the unit disk (recall that polarizations do not depend on adding or subtracting finite-dimensional subspaces). Now we mean that the projection of $W$ to $V^{+}$is a Fredholm operator and the projection of $W$ to $V^{-}$is a smooth operator, i.e. its singular values (considering the Hilbert structures on $W, V^{-}$given by (12) and the analogous form on $V^{-}$) decrease exponentially.

Deciding which maps to call morphisms of open abelian varieties is an interesting problem. For the purpose of the present paper, we will choose morphisms to be only isomorphisms, which is unambiguous.

Definition 2.6. An isomorphism of open abelian varieties

$$
(C, U, S, W, \iota) \rightarrow\left(C^{\prime}, U^{\prime}, S^{\prime}, W^{\prime}, \iota^{\prime}\right)
$$

consists of a bijection $b: C \rightarrow C^{\prime}$, and for each $A \in C$ a bijection $b_{A}: A \rightarrow b(A)$ preserving inbound and outbound boundary components, an isomorphism $\phi: U \rightarrow U^{\prime}$ such that $\phi(W)=W^{\prime}, \phi$ carries $S$ to $S^{\prime}$, and for each system of orders $<$ of all $A \in C$, if we denote by $<_{b}$ the order induced by the system $b_{A}$ on $b(A), b_{A}$ and $\phi$ conjugate $\iota_{<}$to an embedding which defines the same open abelian variety as $\iota_{<_{b}}^{\prime}$. 
In this paper, the category of open abelian varieties will be chosen to be the category whose objects are open abelian varieties and whose morphisms are isomorphisms.

The identifications imposed in Definition 2.5 can be viewed more systematically in the following way: Consider a particular embedding $\iota_{0}: V \rightarrow U$, and a particular hyperbolic basis of $V_{\mathbb{Z}}^{\perp}$. Then we can identify $U$ with $V \oplus V_{\mathbb{R}}^{\perp}$ via this embedding. Now consider the group of all linear transformations

$$
\phi: V \oplus V^{\perp} \rightarrow V \oplus V^{\perp}
$$

which can be represented by $2 \times 2$ matrices

$$
\left(\begin{array}{cc}
\phi_{V V} & \phi_{V V^{\perp}} \\
\phi_{V^{\perp} V} & \phi_{V^{\perp} V^{\perp}}
\end{array}\right)
$$

such that the map $\phi_{V \perp V \perp}$ is an integral symplectic transformation, $\phi_{V V^{\perp}}\left(V_{\mathbb{Z}}^{\perp}\right) \subseteq V_{\text {const }, \mathbb{Z}}, \phi_{V^{\perp} V}\left(V_{\text {deg }, \mathbb{Z}}\right) \subseteq V_{\mathbb{Z}}^{\perp},\left(\phi_{V V}-I d\right)\left(V_{\text {deg }, \mathbb{Z}}\right) \subseteq V_{\text {const }, \mathbb{Z}}$. It is easy to check that linear transformations of this type form a discrete group, which we denote by $S p_{\text {open }}(V, \mathbb{Z})$. This can be considered the group of identifications of open abelian variety data.

More precisely, let us compute the moduli space of open abelian varieties for a given set of open connected components. From the definition, it follows that the moduli space is

$$
U(W) \backslash S p_{\text {sm }}(U) / S p_{\text {open }}(V, \mathbb{Z}) .
$$

The group $U(W)$ is the Hilbert unitary group on $W$, the group $S p_{s m}(U)$ is the real symplectic group of $U$ which when expressed as $2 \times 2$ matrices in the decomposition $W \oplus \bar{W}$, the off-diagonal terms are smooth operators.

It is worth noting that the group $S p_{s m}(U)$ is in fact also contractible, so the moduli space is a " $K(\pi, 1)$-stack". To prove this, by Kuiper's theorem, it suffices to show that the coset space

$$
U(W) \backslash S p_{s m}(U)
$$

is contractible. Expressing the form $S$ as a $2 \times 2$ matrix as discussed above, it is of the form

$$
\left(\begin{array}{cc}
0 & -i I \\
i I & 0
\end{array}\right)
$$

so (16) is isomorphic to the contractible space

$$
\left\{\exp \left(\begin{array}{cc}
0 & A \\
A & 0
\end{array}\right) \mid A \text { is symmetric smooth }\right\} .
$$


Remark 2.7. An open abelian variety with no open connected (and hence no boundary) components is simply a real symplectic space $U$ with integral structure and decomposition

$$
U_{\mathbb{C}}=W \oplus \bar{W}
$$

where $W$ is positive-definite isotropic, in other words, $S_{W \times W}=0$ and $2 i S(x, \bar{x})>0$ for all $x \in W$. This is equivalent data to an abelian variety over $\mathbb{C}$ as in [6].

\section{Gluing and SPCMC structure}

Theorem 3.1. There exists an SPCMC structure on the set of open abelian varieties.

Remark 3.2. Before embarking on this story, let us briefly note the following curious fact: although open abelian varieties model the notion of open connected components, it does not model the notion of closed connected components. Moreover, for the same reason, while one can define genus as one half of the codimension of $V$ in $U$, the structure does not model the genus of an individual open connected component. It is worthwhile pointing out that one can consider a variant of our notion which would keep track of both closed and open connected components, and would be simply a sequence of closed and open abelian varieties with one connected component in our sense. Such structure would also form an SPCMC by our arguments.

The proof of Theorem 3.1 will occupy the remainder of this section. First, note that the stack structure over complex manifolds and coverings follows from the moduli space remarks at the end of the last section. Also, the operation of sum is obvious, realized by direct sum in the obvious sense. So the main point to discuss is gluing.

We have the decomposition

$$
U \cong V \oplus V^{\perp}
$$

where $V^{\perp}$ is the $S$-annihilator of $V$ in $U$. We therefore have a canonical projection given by the decomposition (17)

$$
p: U \rightarrow V .
$$

Composing with the projection $q_{A}$ from $V$ to $V_{A}$ for a connected component $A$, we get a projection

$$
p_{A}: U \rightarrow V_{A} .
$$

Composing further, for $j \in A$, with the projection

$$
q_{A, j}: V_{A} \rightarrow V_{1} / \mathbb{R}
$$


(where $V_{1}$ is the space of real analytic branched functions on $S^{1}$ as in Construction 2.1 and $\mathbb{R}$ is generated by the constants), we get a projection

$$
p_{A, j}: U \rightarrow V_{1} / \mathbb{R} \text {. }
$$

All these maps of course also have complex forms, which we will denote by the same symbol.

Now the idea of gluing an inbound boundary component $i \in A^{-}$to an outbound boundary component $j \in B^{+}, A, B \in C$, is to set

$$
U^{\nabla}=\left\{a \in U \mid p_{A, i}(a)=p_{B, j}(a)\right\} / \operatorname{Im}\left(V_{1}\right) .
$$

Here by $\operatorname{Im}\left(V_{1}\right)$ we denote the image of $V_{1}$ in $U$ by sending an element $x \in V_{1}$ to the sum of $\iota_{<}\left(x_{i}\right)$ and $\iota_{<}\left(x_{j}\right)$ where $x_{i}$ (resp. $\left.x_{j}\right)$ is the same function as $x$ on the $i$ 'th (resp. $j$ 'th) boundary component and zero everywhere else. The order $<$ is selected in such a way that $i$ and $j$ immediately follow each other (see discussion of Cases 1 and 2 below). Then $\operatorname{Im}\left(V_{i}\right)$, by our assumptions, $S$-annihilates $\left\{a \in U \mid p_{A, i}(a)=p_{B, j}(a)\right\}$, so we can choose $S^{\nabla}$ as the form induced by $S$. However, we will need to show that it is a non-degenerate symplectic form. To this end, we will actually first give an independent formula for gluing $W$, and then show that it is compatible with (21).

To glue $W$, we simply take

$$
W^{\nabla}=\left\{a \in W \mid p_{A, i}(a)=p_{B, j}(a)\right\} .
$$

Next, we will define the set of open connected components $C^{\nabla}$ after gluing, which will give us a space $V^{\nabla}$ defined the same way as $V$, with $C$ replaced by $C^{\nabla}$, and an embedding $\iota_{<}^{\nabla}$ after gluing corresponding to a system of orders $<$ before gluing. Of course, one can choose the order $<$, since for different orders the embeddings must be related by composing with a standard transformation. Now there are two principal cases to distinguish:

Case 1: $A=B$. In this case, define $C^{\nabla}$ as the set of components $E^{\nabla}=E$ when $E \neq A \in C$, and $A^{\nabla}=A-\{i, j\}$ provided $A^{\nabla} \neq \emptyset$. Next, assume $i<j<k$ for all $k \in A-\{i, j\}$. Then let the order $<$ after gluing be given by omitting $i, j$. Further, assume that the boundary component corresponding to $i$ is inbound. We define for $x \in V^{\nabla}, \iota_{<}^{\nabla}(x)$ to be the projection of $\iota_{<}(y)$ for any $y=\left(y_{k}\right) \in V$ where $y_{k}=x_{k}$ when $k \neq i, j$, and $y_{i}=y_{j}$ is arbitrary. By definition, this embedding preserves the symplectic form.

Case 2: $A \neq B$. Then $C^{\nabla}$ is the set of $E^{\nabla}=E$ where $A, B \neq E \in C$, and $A^{\nabla}=B^{\nabla}=(A \cup B)-\{i, j\}$, provided $A^{\nabla} \neq \emptyset$. Then assume that $i$ is the greatest element of $A$ and $j$ is the least element of $B$. Assume again that the $i$ 'th boundary component is inbound. Let the ordering on the glued connected component $(A \cup B)-\{i, j\}$ be obtained by juxtaposing the ordering on $A-\{i\}$ before the ordering on $B-\{j\}$. Again, for $x \in V^{\nabla}$, 
we define $\iota_{<}^{\nabla}(x)$ to be the projection of $\iota_{<}(y)$ for any $y=\left(y_{k}\right) \in V$ where $y_{k}=x_{k}$ when $k \neq i, j$, and $y_{i}=y_{j}$ is arbitrary. (Note that in this case, there is a subtlety due to the fact that $x_{k}$ is only defined up to adding two different constants for $k \in A, B$; what we mean is that the difference of the constants is fixed by the requirement $y_{i}=y_{j}$.) Again, we see that this embedding preserves antisymmetric forms.

Remark 3.3. In the Cases 1 and 2 , when $A^{\nabla}=\emptyset$, it simply gets deleted from the data (see comments in the paragraph below Theorem 3.1 at the beginning of this section). It does not affect the rest of the gluing procedure.

It remains to relate the formulas (21), (22), and prove that $S$ remains non-degenerate. First, since we have complete control over the structure of $U^{\nabla}$, it is easy to see that

$$
\begin{array}{ll}
g^{\nabla}=g+1 & \text { in Case 1, } \\
g^{\nabla}=g & \text { in Case 2 }
\end{array}
$$

where $g^{\nabla}$ denotes $1 / 2$ times the codimension of $V^{\nabla}$ in $U^{\nabla}$. Additionally, since $S^{\nabla}$ is induced from $S$ (at least for the particular choice of orderings), we know that $W^{\nabla} \subset U_{\mathbb{C}}^{\nabla}, \bar{W}^{\nabla} \subset U_{\mathbb{C}}^{\nabla}$ are isotropic and $S^{\nabla}$-dual to each other, so in particular

$$
W^{\nabla} \cap \bar{W}^{\nabla}=0
$$

and thus that the natural map

$$
W^{\nabla} \oplus \bar{W}^{\nabla} \rightarrow U_{\mathbb{C}}^{\nabla}
$$

is injective. What remains to be shown is that, viewing (24) as an inclusion,

$$
W^{\nabla}+\bar{W}^{\nabla}=U_{\mathbb{C}}^{\nabla}
$$

or in other words that the map (24) is onto. To show this, we will take advantage of Segal's method [9] of relative dimension. Choosing a polarization of

$$
V_{\mathbb{C}}=V^{+} \oplus V^{-}
$$

compatible with $W$ (for example as discussed in the last section), let

$$
W_{0}=\operatorname{Im}\left(\left.p\right|_{W}\right), \bar{W}_{0}=\operatorname{Im}\left(\left.p\right|_{\bar{W}}\right) .
$$

Denoting relative dimension with respect to the positive space $V^{+}$by $\operatorname{dim}_{V^{+}}$, i.e.

$$
\operatorname{dim}_{V^{+}}(Q)=\operatorname{index}\left(\pi_{Q}\right)
$$

for $Q \subset V_{\mathbb{C}}$ where $\pi_{Q}: Q \rightarrow V^{+}$is the projection given by the decomposition, we get

$$
\operatorname{dim}\left(\operatorname{Ker}\left(\left.p\right|_{W}\right)\right)+\operatorname{dim}_{V^{+}} W_{0}+\operatorname{dim}\left(\operatorname{Ker}\left(\left.p\right|_{\bar{W}}\right)\right)+\operatorname{dim}_{V^{-}} \bar{W}_{0}=2 g
$$


(since $W_{0}$ and $\bar{W}_{0}$ generate $V_{\mathbb{C}}$,

$$
\left.\operatorname{dim}_{V^{+}} W_{0}+\operatorname{dim}_{V^{-}} \bar{W}_{0}=\operatorname{dim}\left(W_{0} \cap \bar{W}_{0}\right)\right) .
$$

But now one has

(28) $\operatorname{dim}\left(\operatorname{Ker}\left(\left.p\right|_{W^{\nabla}}\right)\right)+\operatorname{dim}_{V^{\nabla+}} W_{0}^{\nabla} \geq \operatorname{dim}\left(\operatorname{Ker}\left(\left.p\right|_{W}\right)\right)+\operatorname{dim}_{V^{+}} W_{0}+\epsilon$

where $\epsilon$ is 1 in Case 1 and 0 in Case 2 (this shift arises because of our treatment of the constants on connected components). Equality arises if and only if

$$
W_{0}^{\nabla}+\bar{W}_{0}^{\nabla}=V_{\mathbb{C}}^{\nabla}
$$

Similarly, we have

$$
\operatorname{dim}\left(\operatorname{Ker}\left(\left.p\right|_{\bar{W}^{\nabla}}\right)\right)+\operatorname{dim}_{V^{\nabla}-} \bar{W}_{0}^{\nabla} \geq \operatorname{dim}\left(\operatorname{Ker}\left(\left.p\right|_{\bar{W}}\right)\right)+\operatorname{dim}_{V^{-}} \bar{W}_{0}+\epsilon
$$

$$
\operatorname{dim}\left(\operatorname{Ker}\left(\left.p\right|_{W^{\nabla}}\right)\right)+\operatorname{dim}_{V^{+}} W_{0}^{\nabla}+\operatorname{dim}\left(\operatorname{Ker}\left(\left.p\right|_{\bar{W}^{\nabla}}\right)\right)+\operatorname{dim}_{V^{-}}{\overline{W^{\prime}}}_{0}^{\nabla} \leq 2 g+2 \epsilon .
$$

Comparing (27), (28), (30), (31), we see that equality must arise in (28), (30), so we have (29), which implies (25) by (23) and the comment preceeding (29).

Now integral structure is discussed as follows. First of all, $V_{\mathbb{Z}}^{\nabla \perp}$ is generated by $V_{\mathbb{Z}}^{\perp}$ in Case 2 , and is generated by $V_{\mathbb{Z}}^{\perp}$ and elements which have integral degree on $i$ and differ by an integral value on $i, j$, and have 0 projection to the other boundary components (well defined since we are in the same boundary component) in Case 1 . Such elements must generate $V_{\mathbb{C}}^{\perp}$ by the discussion of the previous paragraph. Additionally, equivalence is preserved by gluing by direct verification.

To define the operations of an SPCMC as defined in [3], we need to soup up our gluing definition to glue simultaneously several pairs of boundary components, each consisting of one inbound and one outbound boundary component.

Regarding the gluing of $U$ and $W$, there are obvious generalizations of formulas (21) and (22) for multiple pairs of components. The trickiest part is the discussion of the ordering of boundary components, since in the case of multiple boundary components, we can no longer rely on distinguishing two cases as we did above. The procedure for generalizing to the case of gluing several pairs is as follows: First, note that for an open abelian variety $\mathfrak{X}$, we can associate an antisymmetric form $S_{<}$with any ordering of the entire set of boundary components of $\mathfrak{X}$, regardless of the open connected components. Simply relate the forms corresponding to the orderings in the standard way, and the embeddings $\iota_{<}$by composing with the standard transformations. (Note that even though the components of an element in 
each open connected component are only defined up to a separate additive constant, this does not affect standard transformations.) For the operation of disjoint union, we simply juxtapose the order (this is possible, as permuting cyclically the boundary components of each disjoint summand does not change the form $S$ ). The general procedure for gluing is to change the order of boundary components (while relating $S$ in the standard way and $\iota_{<}$'s by composing with standard transformations) so that all pairs of boundary components to be glued are arranged so that the outbound component immediately follows the inbound, i.e. the inbound is $i$ 'th and the outbound is $i+1$ 'st, if the boundary components are indexed by integers. The key observation is that permuting $i$ and $i+1$ past another boundary component will not change the value of the form $S$, since the terms of (5) involving $i$ and $i+1$ cancel out, since $f_{i}$ and $f_{i+1}$ are the same function when gluing. Similarly, the standard transformations corresponding to such permutations are identities on functions where $f_{i}$ and $f_{i+1}$ coincide. More generally, embeddings with respect to orders of this specified form which are related by composing with standard transformations before gluing remain related by composing with standard transformations after gluing, since terms coming from the glued boundary components cancel out.

After such arrangement we take the induced embedding $\iota_{<}^{\nabla}$ to be associated with the order $<$ which omits all the pairs of the glued boundary components, and leaves the order of the others unchanged. For a direct definition of the integral structure, $V_{\mathbb{Z}}^{\nabla \perp}$ is generated by $V_{\mathbb{Z}}^{\perp}$ and elements which can be lifted to an element $f$ of the sum of copies of $V_{1}$ over all the boundary components in such a way that $f_{k}=0$ on any boundary component not glued, $f_{i}$ has integral degree and $f_{i}-f_{j}$ is a constant integral function when $i, j$ are glued. We see that this composite gluing produces an open abelian variety, since it will be, for a particular order selected, isomorphic to the open abelian variety obtained by gluing the pairs of boundary components successively.

Next, we must prove that the disjoint union and gluing operations just defined have the coherence isomorphisms and diagrams required in an SPCMC [3].

The coherence isomorphisms correspond simply to the identities required for a commutative monoid with cancellation (Def 3.4 of [3]). The identities are commutativity, associativity, and unitality of sum, unitality and transitivity of cancellation, and distributivity of cancellation under addition. The isomorphisms are by definition determined by what they do on $W$, where sum corresponds to direct sum, and gluing is given by the generalization of (22) to multiple pairs. This is coherent with respect to the obvious maps. 
It is also easy to see that the corresponding maps are compatible with the $\iota_{<}$'s and the integral structure.

Having defined the coherence isomorphisms, we need to consider the commutativity of coherence diagrams. Those diagrams are defined in [3]. All the diagrams required are of the following form: Denote by $X_{a, b}$ the set of open abelian varieties with inbound (resp. outbound) boundary components indexed by the finite set $a$ (resp. $b$ ). Then the basic operations are addition

$$
+: X_{a, b} \times X_{c, d} \rightarrow X_{a+c, b+d}
$$

and unit

$$
0 \in X_{0,0}
$$

(here we denote the disjoint union of finite sets by + , and the empty set 0 , which is the usual notation for commutative monoids with cancellation), and gluing

$$
\nabla: X_{a+c, b+c} \rightarrow X_{a, b}
$$

We consider all words $\mathfrak{W}$ which can be written using $n$ distinct variables $x_{1}, \ldots, x_{n}$, each $x_{i}$ representing an open abelian variety with inbound (resp. outbound) boundary components indexed by $v_{i}$ (resp. $w_{i}$ ). The $v_{i}$ 's and $w_{i}$ 's are in turn words in $m$ variables $a_{1}, \ldots, a_{m}$ (representing finite sets), using the finite set-level operations,+ 0 . No variable $a_{i}$ is allowed to occur more than once among the $v_{i}$ 's, or among the $w_{i}$ 's. However, a variable occuring among the $v_{i}$ 's may also occur among the $w_{i}$ 's (note that otherwise, the operation $\nabla$ could not be applied).

Now coherence diagrams [3] are obtained by the following procedure: Alter a word $\mathfrak{W}$ repeatedly by applying one of the identities (commutativity, associativity, unitality of + , unitality and transitivity of $\nabla$, and distributivity). Denote the word obtained by the end result of this sequence of alterations by $\mathfrak{W}^{\prime}$. Then it is possible that the same word $\mathfrak{W}^{\prime}$ could also be obtained from $\mathfrak{W}$ by a different sequence of alterations. Any time this occurs, we have an obvious corresponding coherence diagram. Our task is to show that all such diagrams commute.

However, this is quite easy, since an isomorphism between open abelian varieties is determined by the isomorphism of the $W^{\prime}$ 's. Now we have a canonical injection

$$
W_{\mathfrak{X}^{\nabla}} \rightarrow W_{\mathfrak{X}},
$$

and also canonical projections

$$
W_{\mathfrak{X}_{1}+\mathfrak{X}_{2}} \rightarrow W_{\mathfrak{X}_{i}} .
$$

Therefore, by induction, we obtain a map

$$
W_{\mathfrak{W}} \stackrel{\phi_{\mathfrak{W}}^{i}}{\longrightarrow} W_{\mathfrak{X}_{i}},
$$


$i=1, \ldots, n$, whose product is injective. By considering all types of coherence isomorphisms again (units, $\nabla$-transitivity, +-commutativity and associativity), we see that the maps (32) and (33) commute with the maps induced by the coherence isomorphisms. Consequently, the two paths $p_{1}$ and $p_{2}$ from the word $\mathfrak{W}$ to the word $\mathfrak{W}^{\prime}$ induce a commutative diagram

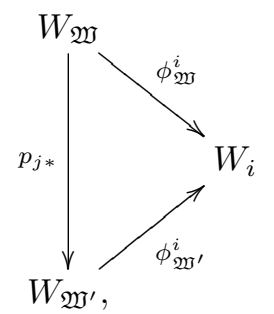

$j=1,2, i=1, \ldots, n$. Since however the product of the maps $\phi_{\mathfrak{W}^{\prime}}^{i}$ is injective, we conclude that $p_{1 *}=p_{2 *}$, as required.

\section{The JaCobian of A WORLDSheET With BOUNDARY}

In this paper, a worldsheet $\Sigma$ is a Riemann surface whose boundary components $c_{1}, \ldots, c_{n}$ are parametrized by analytic diffeomorphisms

$$
\phi_{i}: S^{1} \rightarrow c_{i} .
$$

Taking a chart of $\Sigma$ (and thus identifying with a subset of $\mathbb{C}$ ), boundary components oriented counterclockwise (resp. clockwise) are called inbound (resp. outbound). Worldsheets form an $\operatorname{SPCMC} \mathcal{C}$, as proved in [3].

Theorem 4.1. There exists a morphism of SPCMC's

$$
T: \mathcal{C} \rightarrow \mathcal{J} \text {. }
$$

extending the Torelli map on the moduli stack of closed Riemann surfaces.

We will also call the map $T$ the Torelli map, by extension of the closed case. The proof of Theorem 4.1 will occupy the remainder of this section.

Definition 4.2. A cut worldsheet is a pair $(\Sigma, \Gamma)$ where $\Sigma$ is a worldsheet and

$$
\Gamma \subset \Sigma
$$

is a graph, i.e. a 1-dimensional $\mathrm{CW}$ complex whose edges are piecewise analytic, subject to the two conditions. First, the boundary components $c_{i}$ are required to be edges of $\Gamma$ and $\phi_{i}(1)$ vertices (in particular, the boundary components are not subdivided). Second, the connected components of $\Sigma-\Gamma$ must be surfaces of genus 0 and their number must be equal to the number of the connected components of $\Sigma$. 
Thus, $\Gamma$ basically cuts each connected component of $\Sigma$ into a surface of genus 0 without disconnecting it.

Lemma 4.3. A structure of a cut worldsheet (we will say simply cut structure) exists on every worldsheet.

Proof: Without loss of generality, we can assume $\Sigma$ is connected. To construct $\Gamma$, we can first choose a set of disjoint collectively non-separating curves in $\Sigma$ which cut it to a surface $\Sigma^{\prime}$ of genus 0 , and let the vertices of $\Gamma$ be the images of 0 under the parametrizations. Then connect the vertices by disjoint open edges which cut $\Sigma$ into a disk.

It will be convenient to be a little more specific about the choice of cut structure constructed in the proof of Lemma 4.3. Note that a cut structure on a connected worldsheet specifies a cyclic order of boundary components: changing for the moment the orientation of the boundary components to outbound if necessary, this is simply the order in which the boundary components appear if we travel the boundary of the disk obtained by cutting the worldsheet along $\Gamma$. Now, if $\Sigma$ is connected, we will call $(\Sigma, \Gamma)$ a standard cut structure on $\Sigma$ if the cyclic order of the boundary components of the genus 0 worldsheet $\Sigma^{\prime}$ defined in the proof of Lemma 4.3 is of the form

$$
c_{1}, \ldots, c_{n}, d_{1}, \ldots, d_{2 g},
$$

where $c_{1}, \ldots, c_{n}$ are the boundary components of $\Sigma$, and $\Sigma$ is obtained from $\Sigma^{\prime}$ by gluing $d_{2 i-1}$ with $d_{2 i}, i=1, \ldots, g$. We may refer to the pairs $d_{2 i-1}, d_{2 i}$ as pairs of hidden boundary components of $\Sigma^{\prime}$. A cut structure on a general worldsheet $\Sigma$ will be called standard if its restriction to every connected component of $\Sigma$ is standard.

Now for a Riemann surface with standard cut structure $(\Sigma, \Gamma)$, we define an open abelian variety $T(\Sigma, \Gamma)$ as follows:

Without loss of generality, we may assume that $\Sigma$ is not closed, for in the closed case we just take the ordinary Jacobian. We may further assume that $\Sigma$ is connected, as there is an obvious operation of direct sum on open abelian varieties (as already remarked). Under the assumption, then, there is only one open connected component $A$, and its elements are the boundary components of $\Sigma$. Let, then, $W$ be the space of holomorphic functions

$$
f: \Sigma-\Gamma \rightarrow \mathbb{C}
$$

which extend to holomorphic functions

$$
\tilde{f}: \tilde{\Sigma} \rightarrow \mathbb{C}
$$

such that for every deck transformation

$$
\sigma: \tilde{\Sigma} \rightarrow \tilde{\Sigma}
$$


there exists a number $n_{\sigma, f} \in \mathbb{C}$ such that

$$
\tilde{f}(\sigma z)-\tilde{f}(z)=n_{\sigma, f} \text { for all } z \in \tilde{\Sigma},
$$

factored out by the space of functions constant on each connected component. The space $\bar{W}$ is defined analogously with the word "holomorphic" replaced by "antiholomorphic". Then we must define

$$
U_{\mathbb{C}}=W \oplus \bar{W} .
$$

To define the form $S$ on $U$, first define, for $f \in U$, a 1 -form $\omega_{f}$ on $\Sigma$ by

$$
\omega_{f}=d \tilde{f} .
$$

Then define the ordering $<$ of boundary components as the order in which the boundary components occur on the boundary of $\Sigma-\Gamma$ in the counterclockwise direction. (Recall that only the cyclic order matters.) Then define

$$
S(f, g)=\int_{\Sigma} \omega_{f} \omega_{g}
$$

Lemma 4.4. The restriction

$$
U \rightarrow V_{A}
$$

is onto. More precisely, (39) has a splitting which is canonical on functions of degree 0 on each boundary component, and canonical in the general case subject to selecting a standard cut structure on $\Sigma$.

Proof: Assume without loss of generality that $\Sigma$ is connected and not closed. Recall that by the Dirichlet principle, for a (single-valued) realanalytic function $\phi_{0}$ on $\partial \Sigma$, there exists a unique harmonic function $\phi$ on $\Sigma$ such that

$$
\left.\phi\right|_{\partial \Sigma}=\phi_{0} .
$$

We can then represent uniquely

$$
\phi \in W \oplus \bar{W},
$$

which gives a canonical splitting of (39) on functions of degree 0 . To find a splitting on functions of non-zero degrees, note that, using the notation (37), $c_{1}, \ldots, c_{n}, d_{2}, d_{4}, \ldots, d_{2 g}$ and the paths $p_{1}, \ldots, p_{g}$ on the boundary of the disk $D$ from the vertex $v_{i}$ of $\Gamma$ on $d_{2 i-1}$ and the corresponding point on $d_{2 i}$ form a basis of $H_{1}(\Sigma, \mathbb{Z})$. Therefore, there exists a harmonic form with any given residues along $c_{1}, \ldots, c_{n}$ with sum 0 , and residues 0 along $d_{2}, \ldots, d_{2 g}$, $p_{1}, \ldots, p_{g}$. Integrating the form we obtain a function $\phi$, and subtracting $\phi$ from the original function reduces the general case to the degree 0 case in terms of existence and uniqueness. 
Lemma 4.5. Let $(\Sigma, \Gamma)$ be a genus 0 cut worldsheet and let $<$ be an order of boundary components compatible with the cyclic order specified by the cut. Then, for real analytic functions $f, g$ on $\partial \Sigma$,

$$
S(\tilde{f}, \tilde{g})=S_{A}(f, g)
$$

where $S_{A}$ is the form defined in Construction 2.1, $S$ is (38), and $\tilde{f}, \tilde{g}$ are the harmonic continuations of $f, g$ to the disk obtained by cutting $\Sigma$ along $\Gamma$.

Proof: Let $D$ be the disk obtained from $\Sigma$ by cutting along $\Gamma$. By Stokes' theorem, we have

$$
S(\tilde{f}, \tilde{g})=\int_{D} \omega_{\tilde{f}} \omega_{\tilde{g}}=\int_{\partial D} \tilde{f} d \tilde{g} .
$$

We claim that the right hand side is equal to (5) in the order specified. To see this, we can assume that all the boundary components are outbound, and the graph $\Gamma$ has no vertices except the vertices $v_{1}, \ldots, v_{n}$ on the boundary components $c_{1}, \ldots, c_{n}$, and edges connecting $v_{i}, v_{i+1}, i=1, \ldots, n-1$ (since we can always reach such case by continuous deformation which does not change the value of (41)).

In this case, denoting by $f_{i}, g_{i}$ the restrictions of $f, g$ to $c_{i}$, the contribution to the right hand side of (41) other than from the boundary components $c_{1}, \ldots, c_{n}$ is

$$
\begin{aligned}
& \left(g_{2}(0)-g_{1}(0)-\Delta_{g_{1}}\right) \Delta_{f_{1}}+\left(g_{3}(0)-g_{2}(0)-\Delta_{g_{2}}\right)\left(\Delta_{f_{1}}+\Delta_{f_{2}}\right)+\ldots \\
\ldots+\left(g_{n}(0)-g_{n-1}(0)-\Delta_{g_{n-1}}\right)\left(\Delta_{f_{1}}+\ldots+\Delta_{f_{n-1}}\right) & \\
=- & \sum_{i=1}^{n} g_{i}(0) \Delta_{f_{i}}-\sum_{i \leq j} \Delta_{f_{i}} \Delta_{g_{j}} \\
=- & \sum_{i=1}^{n} g_{i}(0) \Delta_{f_{i}}-\frac{1}{2} \sum_{i=1}^{n} \Delta_{f_{i}} \Delta_{g_{i}}-\frac{1}{2} \sum_{i<j}\left(\Delta_{f_{i}} \Delta_{g_{j}}-\Delta_{g_{i}} \Delta_{f_{j}}\right) .
\end{aligned}
$$

Lemma 4.6. The conclusion of Lemma (4.5) extends to all worldsheets with standard cut structure, provided

$$
\left.\tilde{f}\right|_{d_{2 i-1}}=\left.\tilde{f}\right|_{d_{2 i}} \text { of degree } 0
$$

and

$$
\left.\tilde{g}\right|_{d_{2 i-1}}=\left.\tilde{g}\right|_{d_{2 i}} \text { of degree } 0 \text {. }
$$

Proof: It suffices to assume, without loss of generality, that $\Sigma$ is connected. Then simply apply Lemma (4.5) to $\Sigma^{\prime}$. The additional terms related to $d_{2 i-1}, d_{2 i}$ cancel out. 
Note that the function $\tilde{f}$ in Lemma (4.6) is determined uniquely by $f$ and $\Gamma$. Thus, fixing $\Gamma$, we can now define an open abelian variety $T(\Sigma, \Gamma)$ by choosing $W$ as above, and letting the map (10) be defined by the correspondence $f \mapsto \tilde{f}$. Regarding the integral structure, a function $f \in V^{\perp}$ is integral if all the numbers $n_{\sigma, f}$ are integers. By the proof of Lemma (4.4), this is equivalent to putting

$$
V_{\mathbb{Z}}^{\perp}=\left\{f \in U|f|_{\partial \Sigma}=0, \operatorname{deg}\left(\left.f\right|_{d_{2 i}}\right) \in \mathbb{Z},\left.f\right|_{d_{2 i-1}}-\left.f\right|_{d_{2 i}} \in \mathbb{Z}\right\} .
$$

To show correctness of our definition, it remains to show that $T(\Sigma, \Gamma)$ does not depend on the choice of standard cut structure $\Gamma$. In other words, we need to show that the open abelian varieties constructed by two different choices $\Gamma_{1}, \Gamma_{2}$ of $\Gamma$ are related by conditions (13) and (14). Let us use the same notation as in (13) and (14), with $\iota_{i}, V_{\mathbb{Z}, i}^{\perp}$ constructed from $\Gamma_{i}$. Assume again, without loss of generality, that $\Sigma$ is connected. Looking first at (13), we see from the above comments that for $f \in V_{\mathbb{Z}, 1}^{\perp}$, $d f$ has integral periods with respect to $H_{1}(\Sigma, \mathbb{Z})$ and $f$ has 0 degrees on the boundary components. These conditions do not depend on $\Gamma_{i}$. However, there is an additional condition that the branch of the function $f$ on the disk $D$ obtained by cutting $\Sigma$ along $\Gamma$ has 0 restriction to the boundary components of $\Sigma$. We see that changing the fundamental domain $D$ results in possibly selecting different branches of the function on the boundary components of $\Sigma$, which results in adding an integral linear combination of the periods of $d f$, which are integral constant functions, as claimed.

Regarding (14), we have already shown that the selection of $\tilde{f}$ is canonical in case of $f$ having 0 degrees, so we know (14) in this case. In the general case, again, if $f \in V_{d e g, \mathbb{Z}}$, then $d_{\iota} f$ have integral periods with respect to generators of $H_{1}(\Sigma, \mathbb{Z})$. In addition, the restrictions of $f_{1}$ and $f_{2}$ to the boundary component $c_{j}$ differ at most by selection of a branch (since we use different fundamental domains for calculating the restriction), i.e. by an integral constant function. This proves (14).

To complete the proof of Theorem 4.1, it remains to show that the map $T$ is compatible with gluing. We follow again the two cases of the definition of gluing in the previous section.

Case 1: $A=B$. Assume, without loss of generality, that $\Sigma$ is connected, $\Gamma$ is a standard cut structure on $\Sigma$, and the boundary components are $c_{1}, \ldots, c_{n}$, as in (37). Without loss of generality, then, $\Sigma^{\nabla}$ is obtained from $\Sigma$ by gluing $c_{n-1}$ and $c_{n}$. Then the projection $\Gamma^{\nabla}$ of $\Gamma$ onto $\Sigma^{\nabla}$ defines a standard cut structure on $\Sigma^{\nabla}$, and

$$
T(\Sigma, \Gamma)^{\nabla}=T\left(\Sigma^{\nabla}, \Gamma^{\nabla}\right)
$$

by definition. 
Case 2: $A \neq B$. Without loss of generality, $\Sigma=\Sigma_{1} \amalg \Sigma_{2}$ and we have standard cut structures $\Gamma_{i}$ on $\Sigma_{i}$ such that

$$
\Gamma=\Gamma_{1} \amalg \Gamma_{2},
$$

and the boundary components of $\Sigma_{i}^{\prime}$ are

$$
c_{i, 1}, \ldots, c_{i, n_{i}}, d_{i, 1}, \ldots, d_{i, 2 g_{i}} .
$$

Without loss of generality, further, we are gluing $c_{1, n_{1}}$ to $c_{2,1}$. Then we obtain a standard cut structure $\Gamma^{\nabla}$ on $\Sigma^{\nabla}$ by taking the projection of $\Gamma_{1} \cup \Gamma_{2}$ and omitting the edge corresponding to $c_{1, n_{1}}$ (or equivalently, $c_{2,1}$ ). Again, by definition, we then have (42).

The compatibility of $T$ with disjoint union is obvious, as is compatibility with coherence isomorphisms (the point here, again, being that isomorphisms of open abelian varieties are determined by the isomorphisms of the $W$ 's, so the more subtle structure does not need to be discussed to prove commutativity of diagrams).

\section{The LATtice CONFORMAL FIELD THEORY ON THE SPCMC OF OPEN ABELIAN VARIETIES}

We begin by the same considerations as in [4], starting on p. 351. Consider an even lattice $L$ and a bilinear form

$$
b: L \times L \rightarrow \mathbb{Z} / 2
$$

which satisfies

$$
b(x, x) \equiv \frac{1}{2}\langle x, x\rangle \quad \bmod 2 .
$$

Let $T=L_{\mathbb{C}} / L$. We let $T_{S^{1}}$ denote the space of all real analytic maps $S^{1} \rightarrow T$. We choose a universal cover $T_{S^{1}}^{\prime}$ of $T_{S^{1}}$, which can be considered as a space of maps $[0,1] \rightarrow L_{\mathbb{C}}$. On $T_{S^{1}}^{\prime}$, we have a cocycle

$$
c(\tilde{f}, \tilde{g})=\exp \frac{2 \pi i}{2} \oint_{S^{1}}\left(\tilde{f} d \tilde{g}-\Delta_{\tilde{f}} g(0)+b\left(\Delta_{\tilde{f}}, \Delta_{\tilde{g}}\right)\right)
$$

but $L$ is canonically a normal subgroup of the resulting $\mathbb{C}^{\times}$-central extension $\tilde{T}_{S^{1}}^{\prime}$, so we obtain a canonical $\mathbb{C}^{\times}$-central extension $\tilde{T}_{S^{1}}=\tilde{T}_{S^{1}}^{\prime} / L$,

$$
1 \rightarrow \mathbb{C}^{\times} \rightarrow \tilde{T}_{S^{1}} \rightarrow T_{S^{1}} \rightarrow 1 .
$$

For $\lambda \in L^{\prime} / L$ where $L^{\prime}$ is the dual of $L$, there is now a level 1 Hilbert representation $\mathcal{H}_{\lambda}$ of $\tilde{T}_{S^{1}}$ (its real subgroup acts by unitary bounded operators) distinguished by the fact that the constant subgroup $T \subset \tilde{T}_{S^{1}}$ acts by $e^{2 \pi i\langle ?, \lambda\rangle}$. Our conformal field theory associated with $L, b$ has $L^{\prime} / L$ as its set of labels and $\mathcal{H}_{\lambda}$ as its Hilbert spaces. 
Now consider an open abelian variety $Y=(C, U, S, W, \iota)$. Assume without loss of generality that there is only one open connected component $A$. Consider the pullback

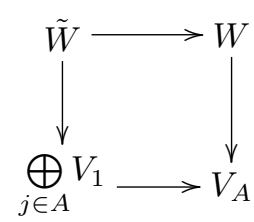

("putting back the constants"). Assuming there is only one connected component, (43) gives a short exact sequence

$$
0 \rightarrow \mathbb{C} \rightarrow \tilde{W} \rightarrow W \rightarrow 0 .
$$

Now let $U_{\mathbb{Z}}^{0} \subset U_{\mathbb{Z}}$ be the sum of $V_{\mathbb{Z}}^{\perp}$ and the lattice spanned by $1_{j} \in V_{0} \cdot j$, $j \in A$. Then

$$
W_{L}=\left\{w \in \tilde{W} \otimes L \mid S(w, u) \in L \text { for every } u \in U_{\mathbb{Z}}^{0}\right\} / L
$$

( $L \subset L_{\mathbb{C}} \subset \tilde{W} \otimes L$ is embedded by the first map (44) tensored with $L$ ). We note that when $Y=T(\Sigma)$ for a worldsheet $\Sigma$, then $W_{L}$ is canonically identified with the space of holomorphic functions $\Sigma \rightarrow T=L_{\mathbb{C}} / L$. Next, we construct a restriction homomorphism

$$
r: W_{L} \rightarrow \prod_{j \in A} T_{S^{1}}
$$

In fact, this map is induced simply by tensoring with $L$ the pullback to $\tilde{W}$ of the projection

$$
r^{\prime}: W \rightarrow V_{\mathbb{C}}
$$

In fact, let us note that we can assume without loss of generality that

$$
\text { (46) is injective. }
$$

Otherwise, $Y$ is a direct sum of $\operatorname{Ker}\left(r^{\prime}\right) \oplus \overline{\operatorname{Ker}\left(r^{\prime}\right)}$ (a closed abelian variety) and its $S$-complement.

Next, note that

$$
\begin{aligned}
& \text { The canonical central extension } \widetilde{\prod_{j \in A} T_{S^{1}}} \text { canonically splits } \\
& \text { when pulled back to } W_{L} \text {. }
\end{aligned}
$$

But in fact, this is completely analogous to the case of surfaces (since the data used there depend only on the Jacobian), which is treated in [4], formulas (58)-(61). Then in the present case, the conformal field theory data 
is given by the space of fixed points

$$
\left(\hat{\otimes}_{j \in A} \mathcal{H}_{\lambda_{j}}^{(*)}\right)^{W_{L}}
$$

for labels $\lambda_{j}, j \in A$ (to simplify notation, the superscript (*) stands for the dual when $j \in A^{-}$and is void when $j \in A^{+}$). Here $\mathcal{H}_{\lambda}, \lambda \in L^{\prime} / L$ are the level 1 irreducible representations of $\tilde{T}_{S^{1}}$. In the case of a closed abelian variety $Y$, the data required are given simply by the space of theta functions on $Y \otimes L$ (see formula (98) of [4]).

The main statement to prove is that the dimension of the space (49) is equal to

$$
\left|L^{\prime} / L\right|^{g}
$$

where $g$ is the genus of $Y$ when we have the condition

$$
\sum_{j \in A} \epsilon_{j} \lambda_{j}=0 \in L^{\prime} / L
$$

where $\epsilon_{j}=1$ resp. -1 when $j$ is outbound resp. inbound, and the dimension of the space (49) is 0 otherwise. To this end, choose a "reference" surface $\Sigma$ of genus 0 (i.e. a disk in $\mathbb{C}$ with a collection of disjoint open disks inside it removed) which has boundary components which match those of $Y$, with opposite orientation. Now the beginning point is that

$$
\bigoplus\left\{\hat{\bigotimes}_{j \in A} \mathcal{H}_{\lambda_{j}} \mid \sum_{j} \epsilon_{j} \lambda_{j}=0\right\}
$$

is contained in the space of sections of the line bundle associated with the principal bundle

$$
\widetilde{\prod}_{j \in A} T_{S^{1}} / \operatorname{Hol}(\Sigma, T)
$$

over

$$
\prod_{j \in A} T_{S^{1}} / \operatorname{Hol}(\Sigma, T)
$$

(In fact, the only reason equality does not occur is convergence issues; a proof follows from the theory of loop groups [7], we do not give the details.)

So this shows that the sum of (49) over $\sum_{j} \epsilon_{j} \lambda_{j}=0$ is contained in (and equal to if we can prove a certain convergence condition) the space of 
sections of the line bundle associated with the principal bundle

$$
W_{L} \backslash \widetilde{\prod_{j \in A}} T_{j} / \operatorname{Hol}(\Sigma, T)
$$

over

$$
W_{L} \backslash \prod_{j \in A} T_{j} / \operatorname{Hol}(\Sigma, T)
$$

But (54) is the closed abelian variety $A$ obtained by gluing $T \Sigma$ to $Y$ tensored with $L$, and (53) is the $\theta$-bundle.

So it remains to show the convergence condition. Again, the method is analogous to [4], Lemma 3. One first uses the boson-fermion correspondence to show the convergence of the "tower modes" of the vacuum operator, i.e. the summand of momentum 0 . Lemma 5 then deals with sum over different momenta. The sum over momenta is treated exactly in the same way in the present case. To discuss the tower modes, there is also bosonfermion correspondence in the category of open abelian varieties. It suffices to discuss the genus 0 case, where on the fermionic side, the vacuum is represented simply by the space $W$ (or more precisely its image in the appropriate Grassmanian). But that element is smooth because we are working in the smooth moduli space.

\section{REFERENCES}

[1] T.M. Fiore: Pseudo limits, biadjoints, and pseudo algebras: categorical foundations of conformal field theory, Mem. Amer. Math. Soc. 182 (2006).

[2] T.M. Fiore: On the cobordism and commutative monoid with cancellation approaches to conformal field theory, J. Pure Appl. Algebra 209 (2007) 583-620.

[3] T.M. Fiore, P. Hu, I. Kriz: Laplaza sets, or how to select coherence diagrams for pseudo algebras, to appear in the Advances of Mathematics.

[4] P. Hu, I. Kriz: Conformal field theory and elliptic cohomology, Adv. Math. 189 (2004) 325-412.

[5] P. Hu, I. Kriz: Closed and open conformal field theories and their anomalies, Comm. Math. Phys. 254 (2005) 221-253.

[6] D. Mumford: Abelian varieties, Tata Inst. of Fund. Res. Studies in Math. 5, London 1970.

[7] A. Pressley, G. Segal: Loop groups, Oxford Math. Monographs, Oxford University Press 1986.

[8] B. Riemann: Theorie der Abelschen Functionen, Journal Reine und Angew. Mathematik 54 (1857) 101-155.

[9] G. Segal: The definition of conformal field theory, Topology, geometry and quantum field theory, London Math. Soc. Lecture Note Ser. 308, Cambridge University Press, 2004, 421-577.

[10] G. Segal: Unitary representations of some infinite-dimensional groups, Comm. Math. Phys. 80 (1981). 301-342. 
Thomas M. Fiore

Department of Mathematics

UNIVERSiTy OF CHICAGO,

5734 S. University, Chicago, IL 60637, USA

AND

Departament de Matemàtiques

Universitat Autònoma de Barcelona

08193 Bellaterra (BARCElona), Spain

E-mail address: fiore@math.uchicago.edu

IGOR KRIZ

Department of Mathematics

UNIVERSITY OF MiCHIGAN

2074 East Hall, 530 Church Street

AnN Arbor, MI 48109-1043, USA

E-mail address: ikriz@umich.edu 